\title{
INTEGRATION METHODS WHERE FORCE \\ IS OBTAINED FROM THE SMOOTHED \\ GRAVITATIONAL FIELD
}

\author{
FRANK HOHL \\ NASA, Langley Research Center, Hampton, Va.
}

\section{Introduction}

Many problems in stellar dynamics involve phenomena occurring in inhomogeneous systems in which the interaction between the particles is fully described by a selfconsistent field operating in phase space. Because the particles interact by means of the long-range Coulomb force, each particle is under the simultaneous influence of a large number of other particles. Therefore, stellar systems will respond to any perturbation in a collective manner, and a study of such systems is concerned essentially with the $N$-body problem.

The collective phenomena do not depend on two-body collisions such as occur in ordinary gases, and therefore the collective effects will be present in collisionless systems. Since the number of particles in the system is large, a distribution function can be used to describe the density of particles in phase space. The distribution function must then satisfy the Vlasov equation (the self-consistent set of the Maxwell equations plus the collisionless Boltzmann equation). In using the Vlasov equation to describe a stellar system the number of masses which make up the system is assumed to become infinite while the total mass remains constant. Although such an approach allows description of the system by means of a distribution function which must satisfy the Vlasov equation, solutions to the time-dependent nonlinear Vlasov (or collisionless Boltzmann) equation are, in general, very difficult to obtain. An attempt is therefore made to condense the large number of stars which a galaxy or other stellar system may contain into a smaller number of superparticles. Numerical or computer models are then used to perform computer experiments simulating Vlasov phenomena by following the simultaneous motion of a large number of superparticles.

Computer models for collisionless systems have been extensively used to study plasmas and plasma flow problems. Much of this work is referred to in the proceedings of the two recent conferences (Symposium on Computer Simulation of Plasma and Many-Body Problems, Williamsburg, Virginia, April 1967, NASA SP-153, and Proceedings of the APS Topical Conference on Numerical Simulation of Plasmas, Los Alamos, New Mexico, Sept. 1968, LA-3990). The application of computer models to collisionless stellar systems has been more recent and we describe below the twodimensional rod model and the model for disks of stars. For these two models the equations of motion used to advance the motion of the stars are the same. The 
essential difference is in the determination of the gravitational field or force acting on a star.

At the present time there are no realistic three-dimensional computer simulations for collisionless systems in progress. The reason for this is simply a matter of economics, that is, limited computer storage and computer time. Nevertheless, the methods for performing three-dimensional calculations are available as a simple extension of the methods used in simulating disks of stars.

\section{Computer Model for Disks of Stars}

One of the more realistic models presently available for simulating stellar systems is the model which simulates the motion of large numbers of stars (point masses) that are confined to move in the plane of a highly flattened stellar system, such as a disk galaxy.

The model effectively simulates the evolution of an isolated disk of stars. Lindblad (1960) pioneered such calculations by following the motion of up to 192 mutually attracting mass points in the given central field of the Galaxy. By placing the mass points initially in a system of concentric rings with circular velocities, Lindblad investigated the mutual disturbances in such a system to simulate the spiral structure of galaxies. Because Lindblad was able to follow the motion of only a rather small number of stars, his model has limited applicability. Miller and Prendergast (1968) developed a model to study the motion of stars in a plane for systems which are doubly periodic and the forces, star positions, and velocities are allowed only discrete (integer) values which are less than some given maximum value.

The two computer models that are now in use for studying the evolution of selfconsistent disk galaxies have recently been described by Miller and Prendergast (1968), and by Hohl and Hockney (1969). With the development of such models it becomes possible to simulate the dynamical evolution of galaxies (Miller, Prendergast and Quirk (1970) and Hohl (1970a, 1970b)) and to check some of the theoretical predictions for stellar systems (Hockney and Hohl (1969)). The dynamics of the gravitational two-stream instability and of the Jeans instability in a plane stellar system have been investigated by Hohl (1970c). Miller (1970) has analyzed in detail some of the discretization properties of plane stellar systems.

\section{A. COMPUTER MODEL}

The computer model used for investigating the dynamics of disk galaxies is illustrated in Figure 1. The $N \times N$ array of cells shown in Figure 1 is superposed over the plane of the galactic disk. The array of cells is introduced only for the purpose of calculating the gravitational potential. The cells are identified by $n, m$ with $n=0,1,2, \ldots, N-1$ increasing in the $x$-direction and with $m=0,1,2, \ldots, N-1$ increasing in the $y$ direction. The cell in the lower left-hand corner is 0,0 and that in the upper righthand corner of the array is $N-1, N-1$. The stars move over this imaginary array of cells. At the center of each cell a mass density is defined which is given by the number 


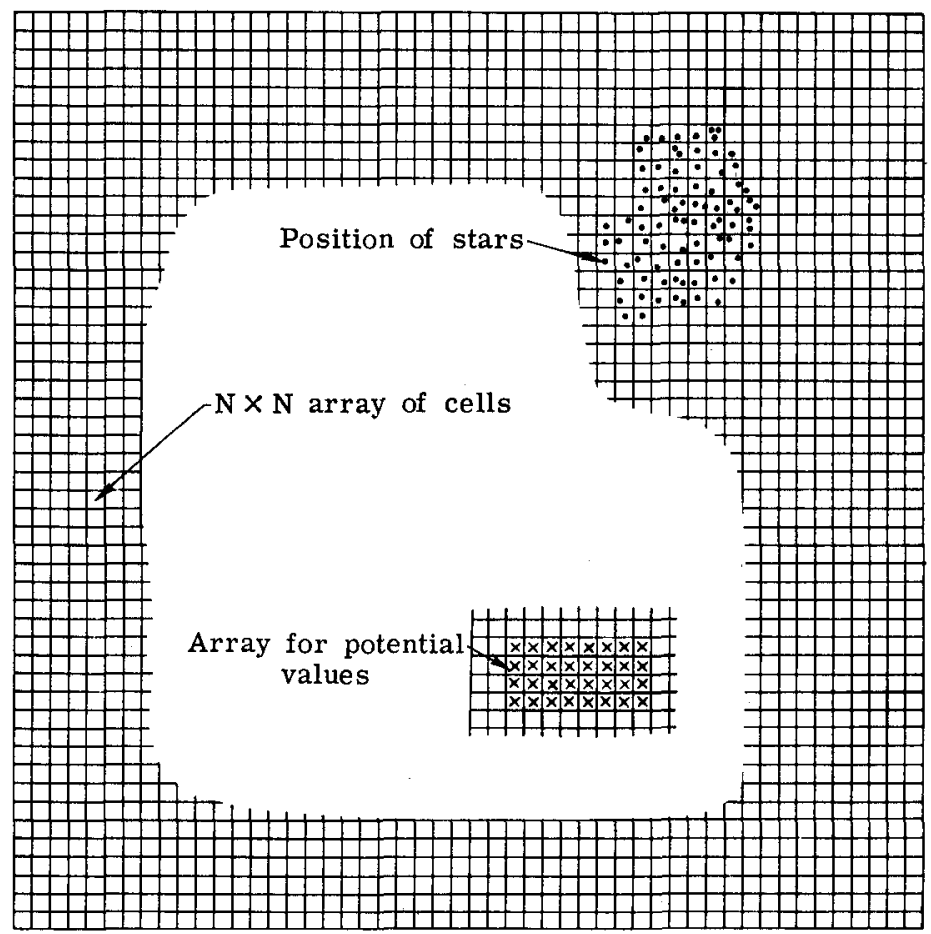

Fig. 1. Computer model illustrating the $N \times N$ array of cells used in calculating the gravitational potential.

of stars in that cell. The number of stars in a cell is usually of the order of 100 and can become much larger near condensations. The density distribution is used to obtain the gravitational potential at the center of each cell. From the gravitational potential, the force acting at the position of a star is computed. Newton's equations of motion are then used to advance the position and velocity of each star by a small time step. For the parameters of a typical galaxy, retardation or relativistic effects need not be considered.

One complete cycle for advancing the motion of the system by a time $\delta t$ consists of the following procedure. First, the distribution of mass $\sigma_{n, m}$ is used to obtain the gravitational potential $\phi_{n, m}$ by effectively summing over the density. Second, the gravitational field at the position of the stars is computed from the potential $\phi_{n, m}$. Third, by applying Newton's laws of motion, the motion of all the stars is advanced for a small time step $\delta t$. This procedure represents one cycle and it is repeated until the desired evolution of the system is achieved.

B. EQUATIONS OF MOTION

The motion of the stars is described by the differential equations

$$
\frac{\mathrm{d} V_{x}}{\mathrm{~d} t}=\frac{\partial \phi}{\partial x} \quad \frac{\mathrm{d} V_{y}}{\mathrm{~d} t}=\frac{\partial \phi}{\partial y}
$$


and

$$
V_{x}=\frac{\mathrm{d} x}{\mathrm{~d} t} \quad V_{y}=\frac{\mathrm{d} y}{\mathrm{~d} t} .
$$

The variable $\phi$ represents the gravitational potential and the gravitational field is given by $K=\nabla \phi$. For a star in the $(n, m)$ th cell, Equations (1) and (2) in the timecentered finite difference form are

and

$$
\frac{1}{\delta t}\left[V_{x}\left(t+\frac{\delta t}{2}\right)-V_{x}\left(t-\frac{\delta t}{2}\right)\right]=\left(K_{x}(t)\right)_{n, m}
$$

$$
\frac{1}{\delta t}[x(t+\delta t)-x(t)]=V_{x}\left(t+\frac{\delta t}{2}\right)
$$

with similar equations for the $y$-components. The numerical calculations can be speeded up greatly by scaling the distance so that the cell dimensions are equal to unity, that is, $\Delta x^{*}=\Delta y^{*}=1$. If, in addition, the velocity and mass of a star are scaled as

$$
V_{x}^{*}=V_{x} \frac{\delta t}{\Delta x}
$$

and

$$
m^{*}=\frac{G(\delta t)^{2}}{2(\Delta x)^{2}} m
$$

then the potential is scaled as

$$
\phi^{*}=\frac{(\delta t)^{2}}{2(\Delta x)^{2}} \phi
$$

The equations of motion take on the simplified form

$$
V_{x}^{*}\left(t+\frac{\delta t}{2}\right)=V_{x}^{*}\left(t-\frac{\delta t}{2}\right)+\left(K_{x}^{*}(t)\right)_{n, m}
$$

and

$$
x^{*}(t+\delta t)=x^{*}(t)+V^{*}\left(t+\frac{\delta t}{2}\right)
$$

Two methods can be used to obtain the gravitational field. The simple method is to let each star in a particular cell experience the same field components, namely,

$$
\left(K_{x}^{*}\right)_{n, m}=\phi_{n+1, m}^{*}-\phi_{n-1, m}^{*}
$$

for the $x$-component of the gravitational field in the $n, m$ cell and

$$
\left(K_{y}^{*}\right)_{n, m}=\phi_{n, m+1}^{*}-\phi_{n, m-1}^{*}
$$

for the $y$-component of the field. Equations (9) and (10) show that all stars in the cell $(n, m)$ experience the same gravitational field and the value of the field will jump 
in crossing the cell boundaries. A smoother variation of the field acting on a star is obtained by means of a bilinear interpolation of the flelds (as given by Equations (9) and (10) at the four cell centers surrounding the position of a particular star. The two components of the field are then given by

$$
\begin{aligned}
K_{x}^{*}= & (1-\delta y)(1-\delta x)\left(\phi_{n+1, m}^{*}-\phi_{n-1, m}^{*}\right)+\delta y(1-\delta x) \times \\
& \times\left(\phi_{n+1, m+1}^{*}-\phi_{n-1, m+1}^{*}\right)+\delta x(1-\delta y)\left(\phi_{n+2, m}^{*}-\phi_{n, m}^{*}\right)+ \\
& +\delta x \delta y\left(\phi_{n+2, m+1}^{*}-\phi_{n, m+1}^{*}\right)
\end{aligned}
$$

and

$$
\begin{aligned}
K_{y}^{*}= & (1-\delta y)(1-\delta x)\left(\phi_{n, m+1}^{*}-\phi_{n, m-1}^{*}\right)+\delta x(1-\delta y) \times \\
& \times\left(\phi_{n+1, m+1}^{*}-\phi_{n+1, m-1}^{*}\right)+\delta y(1-\delta x)\left(\phi_{n, m+2}^{*}-\phi_{n, m}^{*}\right)+ \\
& +\delta x \delta y\left(\phi_{n+1, m+2}^{*}-\phi_{n+1, m}^{*}\right)
\end{aligned}
$$

where the pertinent parameters are defined in Figure 2. It is found that the bilinear interpolation gives a slightly more definite structure for the condensations which occurred during the initial evolution of a system. After about one rotation, the results obtained by the two methods display essentially the same structure.

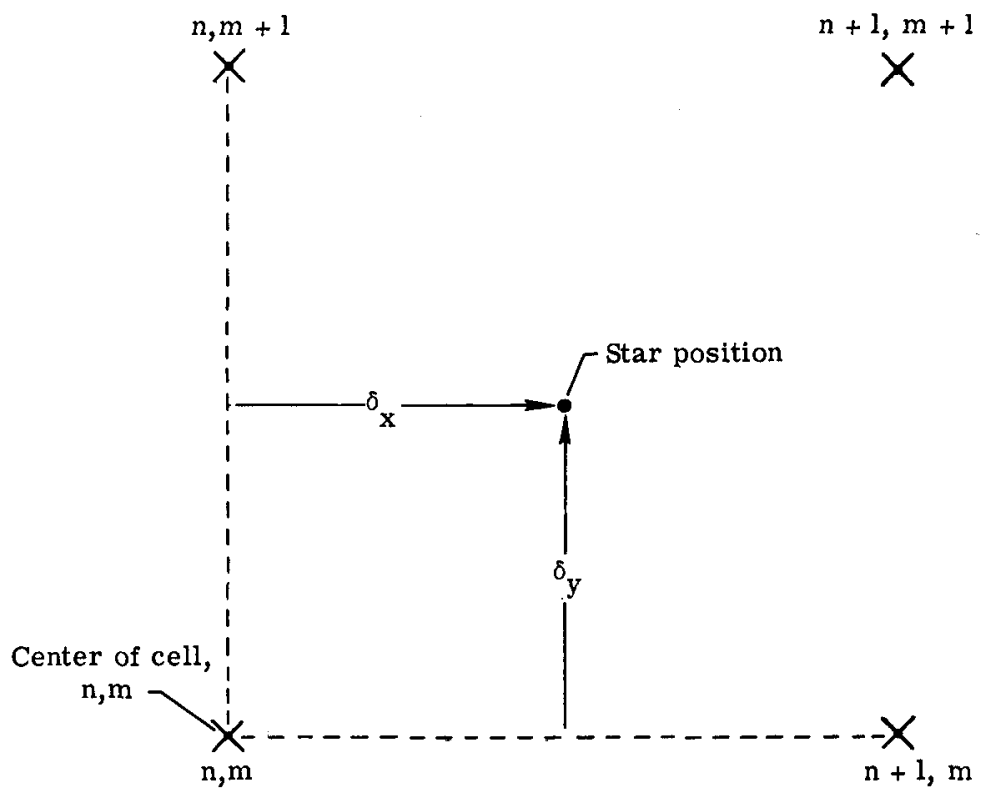

Fig. 2. Parameters used for the bilinear interpolation of the gravitational field.

If a star should leave the $N \times N$ array of cells, the field acting on it is calculated by placing all the mass remaining in the system at the center of the array. The stars outside the array will not interact among themselves, but they will be attracted by the central force due to the mass placed at the center of the array. Whenever an appreciable number of stars leave the array, the calculations are no longer accurate 
and the computer run should be repeated by either increasing the array or by changing the initial conditions.

\section{THE POTENTIAL CALCULATION}

In calculations with the two-dimensional rod model (Hohl, 1968: Hockney, 1967) the gravitational potential is easily obtained by solving the two-dimensional Poisson equation

$$
\frac{\partial^{2} \phi}{\partial x^{2}}+\frac{\partial^{2} \phi}{\partial y^{2}}=4 \pi G \varrho(x, y) .
$$

An attempt therefore might be made to obtain the gravitational potential for the disk model by the same method. The Poisson equation then becomes

$$
\frac{\partial^{2} \phi}{\partial x^{2}}+\frac{\partial^{2} \phi}{\partial y^{2}}+\frac{\partial^{2} \phi}{\delta z^{2}}=4 \pi G \sigma(x, y) \delta(z)
$$

where $\delta(z)$ is the Dirac delta function and $\sigma(x, y)$ is the surface density of stars in the plane of the disk. The difficulty is that no means are available to evaluate $\partial^{2} \phi / \partial z^{2}$. Therefore,

$$
\phi(x, y)=G \iint \frac{\sigma\left(x^{\prime}, y^{\prime}\right)}{\sqrt{\left(x-x^{\prime}\right)^{2}+\left(y-y^{\prime}\right)^{2}}} \mathrm{~d} x^{\prime} \mathrm{d} y^{\prime}
$$

is used to obtain the gravitational potential from the mass density (the primes denote variables over which integration is performed). Presently the density is given only at a finite number of cells separated by a unit distance so that the integral can be changed to a summation

$$
\phi_{n, m}=\sum_{i=0}^{N-1} \sum_{j=0}^{N-1} \sigma_{i, j} H_{i-n, j-m}
$$

where $N$ is the dimension of the array of cells and $H$ is Green's function defined by

$$
H_{i, j}=\frac{1}{\sqrt{i^{2}+j^{2}}} .
$$

To perform directly the summation indicated by Equation (16) requires the summation of $N^{4}$ terms. For $N=100, N^{4}=10^{8}$ and the time required to obtain $\phi$ becomes excessive.

D. FOURIER METHOD

The method to obtain the gravitational potential makes use of the fast Fourier transform methods now available (Cooley and Tukey, 1965). The Fourier transform of the density is defined as

$$
\tilde{\sigma}_{k, 1}=\left(\frac{1}{N}\right)^{2} \sum_{n=0}^{N-1} \sum_{m=0}^{N-1} \sigma_{n, m} \exp \left[i \frac{2 \pi}{N}(k n+l m)\right] .
$$


Similarly, the Fourier transform of Green's function is given by

$$
\tilde{H}_{k, 1}=\left(\frac{1}{N}\right)^{2} \sum_{n=0}^{N-1} \sum_{m=0}^{N-1} H_{n, m} \exp \left[i \frac{2 \pi}{N}(k n+l m)\right] .
$$

Applying the finite convolution theorem to Equation (16) gives the result (Hohl and Hockney, 1969)

$$
\begin{aligned}
\phi_{n, m} & =\sum_{i=0}^{N-1} \sum_{j=0}^{N-1} \sigma_{i, j} H_{i-n, j-m} \\
& =\left(\frac{1}{N}\right)^{2} \sum_{k=0}^{N-1} \sum_{l=0}^{N-1} \tilde{\sigma}_{k, 1} \tilde{H}_{k, 1} \exp \left[-i \frac{2}{N}(k n+l m)\right] .
\end{aligned}
$$

From Equation (22) and the definition of the Fourier transform, it is clear that

$$
\tilde{\phi}_{k, l}=\tilde{\sigma}_{k, l} \tilde{H}_{k, l} .
$$

Therefore, the potential $\phi_{n, m}$ is obtained directly from the inverse Fourier transform of $\tilde{\sigma} \cdot \tilde{H}$. Such a method gives a doubly periodic system. A similar method is used by Miller and Prendergast (1968) in their investigation of doubly periodic stellar systems.

The Fourier transform method just described can be modified to obtain the potential distribution for an isolated system. This modification is achieved by increasing the number of cells by a factor of 4 and by confining the system to one-quarter of the array of cells. The mass density in the remaining three-quarters of the array will then always be identically zero.

Consider now that in addition to the array under investigation, the summation in Equation (16) is extended over all the doubly infinite array of images. However, Green's function $H_{n, m}$ is now modified so that it corresponds to the correct single particle potential for particle separation $r$ less than $N / 2$ (one-half the dimension of the array) and to zero interaction for $r$ greater than $N / 2$. Even though the system is still doubly periodic, there is no longer any interaction between adjacent image systems because their masses are separated by at least $N / 2$.

Thus, to get the correct potential for an isolated system at the expense of a fourfold increase in storage, Green's function to be used in Equation (20) is

$$
\begin{gathered}
H_{n, m}=\frac{1}{\sqrt{n^{2}+m^{2}}} \quad \begin{array}{c}
\left(0 \leqq n, m \leqq \frac{N}{2}\right) \\
\left(n^{2}+m^{2} \neq 0\right)
\end{array} \\
H_{N-n, m}=H_{n, N-m}=H_{N-n, N-m}=H_{n, m}
\end{gathered}
$$

and

$$
H_{0,0}=1
$$


As before, setting $H_{0,0}=1$ is equivalent to setting the self-potential of a star equal to unity. The Fourier transform of $H_{n, m}$ need be done only once. Also, because of the symmetry of $H_{n, m}$ only a finite cosine transform on a $(N / 2+1) \times(N / 2+1)$ mesh is required. The modified Fourier transform approach is described by Hohl and Hockney (1969). It should be pointed out that the Fourier transform method solves Equation (16) for the isolated system exactly (within computer rounding error). It should also be noted that since only one quarter of the $N \times N$ array contains the active potential, the potential calculations can be easily performed such that only storage for an $N \times N / 2$ array is required. A listing of the computer program for obtaining the gravitational potential for isolated disk galaxies is given by Hohl (1970b) and a listing of the fast Fourier transform subroutine is given by Hockney (1970).

The two components of the gravitational field can also be directly computed by the Fourier method by performing the summations

and

$$
\left(K_{x}\right)_{n, m}=-G \sum_{i, j} \frac{\left(x_{n}-x_{i}\right) \sigma_{i, j}}{\left[\left(x_{n}-x_{i}\right)^{2}+\left(y_{m}-y_{j}\right)^{2}\right]^{3 / 2}}
$$

$$
\left(K_{y}\right)_{n, m}=-G \sum_{i, j} \frac{\left(y_{m}-y_{j}\right) \sigma_{i, j}}{\left[\left(x_{n}-x_{i}\right)^{2}+\left(y_{m}-y_{i}\right)^{2}\right]^{3 / 2}} .
$$

However, the computer storage and time required becomes much larger.

E. ARBITRARY FORCE LAW

The method presented for obtaining the gravitational potential can easily be extended to three-dimensional problems. Also, the force law between particles can easily be changed by simply changing Green's function $H$. For example, some of the effects of finite thickness of the galactic disk can be simulated by using finite-length mass rods instead of point masses to represent the stars. The force of attraction $F$ between two mass rods of unit mass and of length $l$ alined perpendicular to the galactic plane with their centers in the galactic plane is

$$
F=\frac{2 G}{l^{2}}\left[\sqrt{1+\left(l^{2} / r^{2}\right)}-1\right]
$$

where $r$ is the separation of the two rods. Green's function then becomes

$$
H_{n, m}=\frac{2}{l}\left\{\begin{array}{r}
r_{n, m} \\
l
\end{array}\left[1-\sqrt{1+\left(l^{2} / r_{n, m}^{2}\right)}\right]+\log _{e}\left[\frac{l}{r_{n, m}}+\sqrt{1+\left(l^{2} / r_{n, m}^{2}\right)}\right]\right\}
$$

where $r_{n, m}=n^{2}+m^{2}$.

\section{F. CALCULATION OF ENERGY AND MOMENTUM}

The angular momentum of the disk at time $t$ is given by

$$
\Gamma(t)=\sum_{i} m_{i}\left[x_{i} \bar{V}_{x, i}-y_{i} \bar{V}_{y, i}\right]
$$


where $m_{i}$ is the mass of the $i$ th star, $\bar{V}_{x, i}=\left[V_{x, i}(t+\delta t / 2)+V_{x, i}(t-\delta t / 2)\right] / 2$ is the $x$-component of the velocity of that star, and the summation extends over all the stars.

The kinetic energy of the disk at time $t$ is

$$
T(t)=\frac{1}{2} \sum_{i} m_{i}\left[\bar{V}_{x, i}^{2}+\bar{V}_{y, i}^{2}\right]
$$

An approximate expression for the potential energy is

$$
P=-\frac{1}{2} \sum_{n} \sum_{m} \sigma_{n, m} \phi_{n, m}
$$

A better definition of the potential energy should be devised by using the definition of potential energy as the work done on a test star and apply it to the present model. The difficulty in the use of Equation (27) is that in regions of large mass condensations this equation gives a value of $\phi$ which is too large for the potential energy.

\section{Two-Dimensional Rod Model}

Computer simulations of 'Cylindrical Galaxies' by means of two-dimensional mass rod models have been performed by Hockney $(1967,1968)$ and by Hohl $(1968,1969)$. The stability and dynamics of systems containing up to 100000 mass rods were investigated. However, the rod-star approximation is likely to be valid only for very few galaxies, such as possibly NGC 2685. The model is essentially identical to that for disk galaxies except that the gravitational potential is now strictly two dimensional and is obtained by solving the two-dimensional Poisson equation. The gravitational potential $\phi_{n, m}$ is then obtained from the density $\varrho_{n, m}$ by solving the two-dimensional Poisson equation

$$
\frac{\partial^{2} \phi}{\partial x^{2}}+\frac{\partial^{2} \phi}{\partial y^{2}}=4 \pi G \varrho(x, y)
$$

by finite difference methods. The standard five-point difference equation

$$
\phi_{n+1, m}+\phi_{n, m+1}+\phi_{n-1, m}+\phi_{n, m-1}-4 \phi_{n, m}=4 \pi G \varrho_{n, m}
$$

is generally used to solve for the potential distribution. The cell dimensions $\Delta x$ and $\Delta y$ are taken to be equal to unity.

The potential at the boundary of the rectangular region is required in the solution of the Poisson equation. At an arbitrary boundary point a distance $z=x+i y(i=\sqrt{-1})$ from the center of the mesh, the potential is given by

$$
\begin{aligned}
\phi(z) & =2 G \sum_{n, m} \varrho_{n, m} \log _{e}\left|z-z_{n, m}\right|= \\
& =2 G M \log _{e}|z|+2 G \sum_{n, m} \varrho_{n, m} \operatorname{Re}\left|\log _{e}\left(1-\frac{z_{n, m}}{z}\right)\right|
\end{aligned}
$$

where $M$ is the total mass in the system and $z_{n, m}=x_{n, m}+i y_{n, m}$ is the coordinate of 
the cell $n, m$. Since $\varrho_{n, m}$ is nonzero only for $z_{n, m} / z<1$, Equation (30) can be written as

where

$$
\phi(z)=2 G M \log _{e}|z|-2 G R e \sum_{k} \frac{a_{k}}{k z^{k}}
$$

$$
a_{k}=\sum_{n, m} \varrho_{n, m} z_{n, m}^{k}
$$

and the series expansion for $\log _{e}\left(1-z_{n, m} / z\right)$ is truncated after 15 terms.

The set of simultaneous equations given by Equation (29) can be solved by an iteration of the form

$$
\begin{aligned}
\phi_{n, m}^{r+1}=\phi_{n, m}^{r}+\gamma\left(\phi_{n-1, m}^{r+1}+\phi_{n+1, m}^{r}\right. & +\phi_{n, m-1}^{r+1}+ \\
& \left.+\phi_{n, m+1}^{r+1}-4 \phi_{n, m}^{r}-4 \pi G \varrho_{n, m}\right) .
\end{aligned}
$$

For the purpose of saving computer storage and increasing the convergence rate, the new values of $\phi$ (that is, $\phi^{r+1}$ ) which have already been calculated during a particular iteration are used in the right-hand side of Equation (33). The superscript $r$ refers to the $r$ th iteration and the parameter $\gamma$ is adjusted to give the maximum rate of convergence.

If the motion of all the stars in the system is advanced for a small time step $\delta t$, the mass distribution $\varrho_{n, m}$ will not change very much. The change in the gravitational potential will then also be very small. Thus, the solution of the finite difference form of the Poisson equation (Equation (33)) by an iteration method which uses the potential from the previous cycle as an initial guess will converge very rapidly. The accuracy of the iterative solution of the Poisson equation is easily checked during the calculations. This verification is made by obtaining the values of the potential and the field at several selected points by summing directly the contribution from each star. The values so obtained agree with those obtained from the solution of the Poisson equation to at least the first three digits. The number of iterations required for a $51 \times 51$ mesh was found to be 5 to 7 and for a $101 \times 101 \mathrm{mesh}, 12$ iterations (Hohl, 1969).

The initial guess of the potential at $t=0$ is determined by using analytical expression for the potential of the initial cylinder (Hohl, 1969).

Since direct methods for solving the set of Equations (29) are now generally available it is preferable to use them for obtaining the potential.

The method described previously for disks of stars can of course also be used for obtaining the potential for the two-dimensional rod model. However, the method of solving the Poisson equation is faster and requires less computer storage.

\section{Summary}

The dynamics of collisionless stellar systems can be studied by representing the system by large numbers of representative stars. The numerical methods that are 
used to integrate the motion of the system in time are presented in some detail. Examples of actual computer experiments can be found in the literature cited.

\section{References}

Cooley, J. W. and Tukey, J. W.: 1965, Math. Comput. 19, 297.

Hockney, R. W.: 1967, Astrophys. J. 150, 797.

Hockney, R. W.: 1968, Publ. Astron. Soc. Pacific 80, 662.

Hockney, R. W.: 1970, in Methods in Computational Physics, 9, 135, Academic Press Inc., New York. Hockney, R. W. and Hohl, F.: 1969, Astron. J. 74, 1102.

Hohl, F.: 1968, Bull. Astron. 3, 227.

Hohl, F.: 1969, 'Computer Simulation of a Cylindrical Galaxy', NASA TN D-5200.

Hohl, F.: 1970a, in W. Becker and G. Contopoulos (eds.), 'The Spiral Structure of our Galaxy', I.A.U. Symp. 38, 368.

Hohl, F.: 1970b, 'Dynamics of Disk Galaxies', NASA TR R-343.

Hohl, F.: 1970c, Astron. J. 76, 202.

Hohl, F. and Hockney, R. W.: 1969, J. Comput. Phys. 4, 306.

Lindblad, P. O.: 1960, Stockholm Obser. Ann. 21, 3.

Miller, R. H.: 1970, J. Comput. Phys. 6, 449.

Miller, R. H. and Prendergast, K. H.: 1968, Astrophys. J. 151, 699.

Miller, R. H., Prendergast, K. H., and Quirk, W. J.: 1970, Astrophys. J. 161, 903. 